\title{
ENSEÑANZA DEL ÁLGEBRA DE VECTORES CON ENFOQUE POR COMPETENCIAS A IMPLEMENTARSE EN FÍSICA DE EDUCACIÓN SECUNDARIA
}

\author{
Abner Ahnet Barrera Fernández ${ }^{1}$ \\ Nicolás Enrrique Rivas Báez ${ }^{2}$ \\ William Oswaldo López Flores ${ }^{3}$
}

\section{Resumen}

Esta investigación ha caracterizado el proceso de enseñanza del Álgebra de Vectores implementada en Física desde la perspectiva de la Educación Matemática en la enseñanza de Educación Secundaria, sus características y dificultades en la enseñanza. El enfoque es cuantitativo con un diseño descriptivo. La muestra la conformaron 97 estudiantes del décimo grado y 4 docentes de las asignaturas de Física y Matemática, del Instituto Nacional Autónomo "Rubén Darío", del municipio de Nueva Guinea, a los cuales se les aplicaron test, encuesta, entrevista y guía de observación. En el análisis se ha utilizado el Sistema Estadístico para la Ciencias Sociales (SPSS), en los resultados de relevancia se puede mencionar el uso inadecuado de las propiedades de Álgebra de Vectores durante los procedimientos aplicados a definiciones y conceptos físicos por los estudiantes; asimismo, la búsqueda de los docentes para contrarrestar las debilidades en el aprendizaje del álgebra y la unificación de la herramienta Matemática de manera significativa, en el programa de estudio de Física de Educación Secundaria.

Palabras clave: Instrucción Matemática, Álgebra de Vectores, Física, Matemática, Enseñanza y Currículo.

\section{Summary}

This research has characterized the teaching process of linear algebra implemented in physics from the perspective of mathematics education in Secondary school education, their educational characteristics and difficulties. The approach is quantitative with a descriptive design. The sample consisted of 97 students from the tenth grade and 4 teachers that teach physics and mathematics at the National Autonomous Institute "Ruben Dario", of the municipality of Nueva Guinea to whom they applied, test, polls, interview, and observation guide. The statistical system for social sciences (SPSS) was used in the analysis, amount the relevant results, we can mentioned misuse of the properties of linear algebra during procedures applied to definition and concepts of physics by the students, likewise the Search for teachers to counteract the weaknesses

\footnotetext{
Licenciado en Ciencias de la Educación con Mención en Matemática, barrera.abner@gmail.com.

Licenciado en Ciencias de la Educación con Mención en Matemática, rivasnicolas33@yahoo.com.

Máster en Investigación en Didácticas de las Ciencias Experimentales y Matemática, Gestor de Proyectos de la Dirección de Cooperación Externa de la URACCAN, william.flores@uraccan.edu.ni
} 
in the learning of algebra and the unification of the mathematic tools in a significant way in the physics programme studies of the Secondary Education.

Keywords: Mathematic Instruction, Linear Algebra, Physics, Mathematics, education and curriculum.

\section{Introducción}

El artículo presenta el Álgebra Vectorial, como ente abstracto del conocimiento Matemático, y se sustenta en ideas que tienen que ver con la Física. Este conocimiento matemático se oferta a estudiantes de diferentes niveles y sistemas educativos para el desarrollo del pensamiento científico, fomentando el aprendizaje próximo a temáticas como el cálculo y otras asignaturas de carácter numérico en los niveles de Educación Superior. Según el currículo (2003-2009) del sistema educativo nicaragüense, el desarrollo del álgebra de vectores, en las aulas de clases, era punto de discusión entre las Matemática Elemental y Física Interactiva. En la actualidad, el Ministerio de Educación Nicaragüense (MINED), introduce esta unidad en el área de Física, donde la metodología requiere la fundamentación teórica, práctica y amplio dominio de análisis matemáticos por el docente, para respaldar y dar explicación a los fenómenos que ocurren en el medio, debido a la complejidad misma del contenido y sus aplicaciones.

Desde esta óptica, la preocupación es pedagógica, ya que el objetivo de la educación matemática es mejorar el aprendizaje de los estudiantes, por esta razón, se facilita a los estudiantes un conjunto de conocimientos, con los cuales puedan estructurar nuevos conceptos, idóneos para explicar fenómenos y aplicar procedimientos para dar solución a problemáticas en el medio, por ello, para ayudar a los estudiantes, con las dificultades que se le presenten, esta investigación se centró en describir algunas características del proceso de enseñanza del Álgebra de los Vectores en la asignatura de Física y que esto permite solucionar dificultades que enfrentan los educadores en el desarrollo de la unidad aplicada en la asignatura de Física.

\section{Revisión de literatura}

Se considera que fue Hamilton quien introdujo el término Vector, del latín Vector; que transporta. Esta noción ya había sido manejada "explícitamente por Matemáticos desde la época griega" según Etayo \& Cols. (1995, pp 74-75), por lo tanto hablar de Vectores en aplicaciones de Física, es utilizar y hablar de Matemática Elemental, como herramienta para soportar conceptos físicos de diferentes estados algebraicos, en los que incide el uso de unidades de medidas, por ejemplo; en el, he aquí la versión más sencilla, llamada cantidad, entre las que se han definido operaciones con Vectores y en la cual se clasifican dos tipos de cantidades; escalares (son cantidades que solo tienen magnitud) y vectoriales, donde un Vector describe un evento con una magnitud en una dirección en el espacio. 
En el año 2009 el sistema educativo nicaragüense, adquiere una corriente denominada: Educación basada en competencias, que Lupiañez y Rico (2005) caracterizan como un "sistema educativo que enfatiza la especificación, aprendizaje y demostración de aquellas competencias (conocimientos, destrezas y actitudes) que tienen una importancia central para determinadas tareas" (Lupiañez y Rico 2005, pp.37-38). No muy alejada de las competencias adquiridas por los estudiantes en la disciplina de Matemática, se pretende en la disciplina de física que los estudiantes sean capaz de graficar, determinar componentes y Vectores resultantes, haciendo uso del Álgebra de Vectores aplicada a Física, para lograr estos indicadores el Ministerio de Educación Nicaragüense (MINED), aclara que la disciplina de Física "Se apoya en el método científico, los avances tecnológicos, el razonamiento crítico, reflexivo, creativo e innovador, para tener una visión amplia del mundo que le rodea, a partir de lo práctico, experimental y aplicable" (MINED, 2009, p. 62).

En este sentido, se vislumbra la necesidad del conocimiento conceptual, la aplicación y experimentación de esta disciplina, tomando en cuenta los procesos matemáticos elementales y la búsqueda de formas para aproximarla a la realidad de los estudiantes para darle una explicación a las definiciones adquiridas en el aula de clases, y las cuales coaccionan con la necesidad de los docentes en propiciarle a los estudiantes las herramientas básicas, para construir estos conceptos desde el enfoque experimental y teórico, más aun cuando estas contienen herramientas Matemáticas, como método analítico para fundamentar la solución o explicación de un fenómeno predefinido. En Moreno (2000) se señalan cuatro aspectos que se deben considerar dentro de la resolución de problemas como estrategia didáctica, para la enseñanza por medio de la resolución de problemas, los cuales son:"Enseñar a resolver "problemas tipos". Inducir la reformulación verbal del problema a resolver. Facilitar por medio de preguntas el análisis del enunciado del problema. Facilitar la explicación de los razonamiento presentes durante el proceso de resolución del problema" (Moreno, 2000, p. 30).

Estando consciente de las necesidades y las competencias que exige el currículo, la metodología aplicada debe buscar la forma de estructurar los conceptos y de ajustar los fenómenos ocurridos en el medio, de tal forma que el estudiante sea capaz de interactuar con ellos, partiendo del origen de las situaciones, hasta la parte medular donde se apliquen los conceptos matemáticos abordados en los contenidos, que permitan describir las características del Álgebra de Vectores, desde un punto de vista global y su aproximación a los niveles elementales de la Física contemporánea, de forma objetiva y critica, los cuales contribuyan a la investigación de situaciones del aprendizaje, apropiándose de nuevos conceptos y reconstruyendo de forma constante lo ya adquiridos por medio de la experiencia.

Lupiáñez y Rico (2006) se plantean que "Si el objetivo es que los escolares lleguen a desarrollar ciertas capacidades, ese adiestramiento ha de mostrarse en la ejecución de tareas que muestren de qué son o no capaces esos escolares" (Lupiáñez y Rico, 2006, p. 
40). Por eso, las tareas que se planteen a los estudiantes han de tener en cuenta el uso del conocimiento Matemático al delimitar las capacidades qué se esperen desarrollar en ellos y como docentes, nuestra meta estará centrada en lograr que los estudiantes tomen conciencia de lo que en realidad se está hablando, desde la modelización abstracta de la Matemática, hasta la interrelación de los Conceptos Matemáticos con definiciones en diferentes campos de estudio, siendo capaz de caracterizar los conceptos que inciden en la problemática de estudio, para así aplicar procedimientos eficaces para cada caso en los cuales incurra el análisis de conceptos modelados en el medio en este caso la metodología aplicada se centrara en : establecer, analizar y organizar las actividades y competencias, que el docente espera desarrollar en los estudiantes en torno al tema Matemático, siendo fundamental la auto-preparación teórica-práctica de conceptos y estrategias metodológicas aplicadas, que propicien el desarrollo eficaz y significativo del contenido, por medio de la coacción entre las partes involucradas y el contexto en el que se piensa aplicar.

\section{Materiales y métodos}

El enfoque de este estudio es cuantitativo, está metodología ayudó al tratamiento de los datos a través de la categorización y descripción de las propiedades, características y los perfiles de las personas, grupos, comunidades educativas, procesos y objetos o cualquier otro fenómeno que se sometió al análisis (Hernández, Fernández, \& Baptista, 2010). Mientras que su diseño es descriptivo por que tiene como objetivo indagar la incidencia de las modalidades o niveles de una o más población. Por estas razones, se utilizaron los instrumentos tales como: test, encuesta, entrevista, el diálogo formal y la observación. En esta investigación, la muestra se estimó mediante el programa STATS, donde se estableció un nivel de confianza del $95 \%$, un porcentaje estimado de la muestra de $88 \%$, un margen de error del $5 \%$ aplicado a un tamaño de la población de 110 estudiantes se obtuvo una muestra de 97 estudiantes del décimo grado. En el caso de los profesores, se entrevistaron a los docentes de la asignatura de física (4 profesores).

La combinación de técnicas permitió realizar una triangulación de datos, donde se obtuvieron las descripciones y conclusiones, las cuales accedió al análisis intensivo a través de múltiples fuentes de datos, la construcción de explicaciones integradas por medio de la triangulación de datos y soportada por la revisión de temáticas relevantes. Por lo que la entrevista, la observación, el dialogo formal y el test se analizaron a través de una matriz conceptualmente ordenada donde sus columnas agrupan los ítems que aparecen agrupados conceptual o empíricamente. Mientras que la encuesta se procesó a través de análisis estadístico descriptivo. 


\section{Resultados y discusión}

\section{Características del proceso de enseñanza del Álgebra de los Vectores en la Asignatura de Física de décimo grado}

Con respecto a la disciplina de Física, en la Educación Secundaria, es desarrollada por un docente cuya preparación profesional no es de Física, debido a que el centro no cuenta con un profesional en la disciplina, la asignatura es atendida por profesores de Matemática, los cuales cumple una carga horaria en los diferentes niveles de Educación Media, entre las cuales 12 horas clase (45 minutos/hora clase) corresponden a la disciplina de Física en los turnos vespertino y matutino.

Según el criterio de los profesores de Matemática, así como también los de docentes de Física, la unidad: magnitudes escalares y vectoriales, como se nombra en el programa de Física de décimo grado, pertenece a Matemática y se le llama Álgebra vectorial, en cambio para el 79\% de los estudiantes, la unidad de Magnitudes escalares y vectoriales pertenecen a la Física y explican que: (según los estudiantes 7, 16, 48, 59) "Tiene relación el contenido con la Física debido a que se ven procedimientos con los Vectores, componentes regulares, sumas, restas, representar de forma gráfica, además de aplicar a los problemas de la Física, mientras se encuentran cosas no se observan a simple vista". En cambio un $11 \%$ de los estudiantes explica que la unidad no pertenece a Física debido a que el tema en ocasiones se relaciona a la Física un poco, no mucho, y por lo general parece más Matemática, además el docente utiliza ejercicios de Matemática y es difícil entenderle a las aplicaciones cuando se trata de Física. El restante, es decir, el 10\% de los estudiantes no respondieron a la pregunta, por lo que se considera que para dicho porcentaje el contenido de magnitudes no es significativo o carece de una explicación hacia donde se dirige dicha unidad.

Los docentes aclaran que "la relación del álgebra vectorial con la Física, radica en que la ciencia inmediata en la que tiene aplicaciones es la Física, debido a que también se les conoce como magnitudes vectoriales cuando se habla de estática de sólidos" pero recomiendan que "Lo idóneo sería que se impartan en ambas disciplinas, ya que orienta a la construcción geométrica, basada en la realidad de la naturaleza donde se desarrolla el estudiante".

\section{Contenidos impartidos por los Profesores y la integración de las tecnologías de Información y Comunicación en la enseñanza del Álgebra Vectorial}

La gráfica No. 1 muestra los contenidos recibidos por los estudiantes, en la cual el $84 \%$ a nivel general aseveran haber recibido como contenido los componentes de un Vector, el $75 \%$ de los estudiantes expresan haber recibido operaciones con Vectores (suma, resta, multiplicación), así como también, operaciones con Vectores y escalares, 


\section{EDUCACIÓN}

por último el 28\% de los estudiantes afirma haber recibido operaciones con Vectores aplicados a problemas de Física:

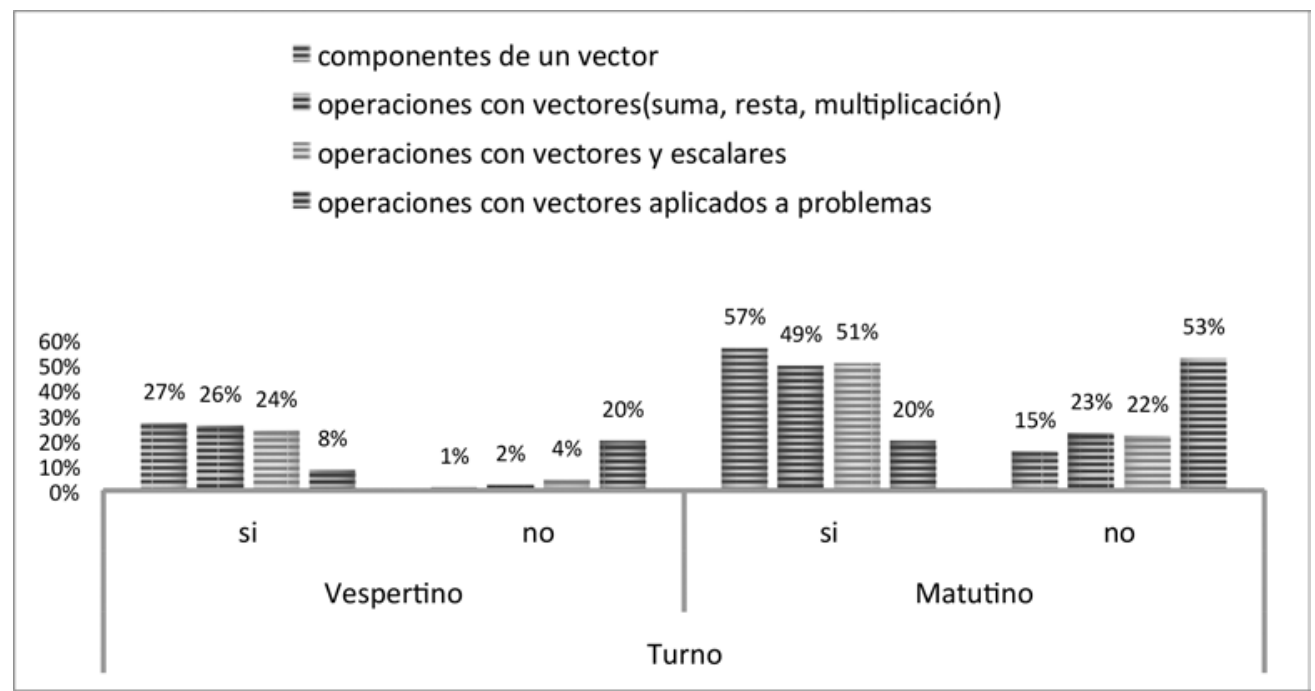

Figura No. 1: Contenidos impartidos por el docente a los estudiantes de décimo grado en la asignatura.

Caracterizando la sistematización de los contenidos impartidos por el docente, se estima que el $20,8 \%$ se centra en el estudio de los componentes de un Vector, el 18\% se dedica al estudio de operaciones con Vectores (adición, sustracción, multiplicación de Vectores), el 18,5\% se ajusta al estudio de operaciones con Vectores y escalares, por ultimo las operaciones con Vectores aplicado a problemas de Física se les brinda un 6,9\% en contrariedad con los docentes de Matemática quienes expresan que el desarrollo del contenido se adecuen al medio donde interactúa el estudiante de forma integral, promoviendo la solución de situaciones y aplicaciones donde el contenido se transforme de lo abstracto a la realidad del entorno del estudiante, véase en el cuadro No. 1.

Cuadro No. 1: Contenidos Impartidos por el docente durante el año lectivo

\begin{tabular}{|l|r|r|r|r|r|}
\hline \multirow{2}{*}{ Contenidos impartidos por el docente durante el periodo lectivo } & \multicolumn{4}{|c|}{ Turno } & \multirow{2}{*}{ Total } \\
\cline { 2 - 5 } & \multicolumn{2}{|c|}{ Vespertino } & \multicolumn{2}{c|}{ Matutino } & \\
\cline { 2 - 5 } & \multicolumn{1}{|c|}{ Si } & \multicolumn{1}{c|}{ No } & \multicolumn{1}{c|}{ Si } & \multicolumn{1}{c|}{ No } & \\
\hline Componentes de un vector & 26 & 1 & 55 & 15 & 97 \\
\hline Operaciones con vectores(suma, resta, multiplicación) & 25 & 2 & 48 & 22 & 97 \\
\hline Operaciones con vectores y escalares & 23 & 4 & 49 & 21 & 97 \\
\hline Operaciones con vectores aplicados a problemas & 8 & 19 & 19 & 51 & 97 \\
\hline Total & 82 & 26 & 171 & 109 & 388 \\
\hline
\end{tabular}


Los materiales más utilizados por los docentes en clases y los que mejores resultados han brindado durante el desarrollo de la unidad son los elementos del estuche geométrico y el uso de la calculadora científica, pero que "para desarrollar el carácter abstracto así como explicar el de traslaciones, han utilizado en ciertas ocasiones de las TIC para presentaciones en Power Point en el aula de clases, debido a que los estudiantes carecen de la imaginación necesaria para hacer traslaciones y operaciones en este nivel" (docente de Física).

\section{Las visitas o sesiones de clases en el aula TIC}

El 83\% de los estudiantes expresan que en este año de clases, "hasta la fecha no recuerdan haber visitado el aula TIC'S o de computación" (Estudiante 21), como ellos le llaman, a diferencia del 14,46\% que asegura haber visitado en una ocasión el aula TIC con el docente para realizar algún tipo de investigación con respecto al tema. Lo que significa que el docente de Física ha utilizado elementos de las TIC para el desarrollo del contenido en el aula de clases (data show, ordenador), pero el estudiante no ha hecho uso personal de estos elementos, lo que convierte la clase a un modelo tradicional con elementos TIC como medios visuales, obviando la verdadera importancia del aprendizaje por medio de la experimentación, del ensayo error. A lo que docentes de Física agregan que" no ha utilizado ningún software educativo de Matemática y Física, para explicar Vectores libres en el espacio", quedando demostrado la inutilidad de las TIC, si el docente no se percata de la necesidad para mejorar el aprendizaje en nuestros estudiantes utilizando los conocimiento y elementos que pueden proporcionar la TIC'S como aula.

En cuanto a la utilización de software educativo, por parte de los estudiante, se asegura que en la asignatura de Física, no se ha utilizado ningún tipo de programa educativo o juegos didácticos, a lo que uno de los docentes de Matemática expone: "para explicar Vectores libres en el espacio le ha sido más útil el programa de escritura WORD, aclarando que los estudiantes presentan dificultades en el uso adecuado del ordenador", a pesar de esto, 6 estudiantes, que representan el 6,1\% de la muestra, del turno vespertino y 34 estudiantes del turno matutino, que representa el $35 \%$ de la muestra, consideran que los programas (software educativo) les serán de utilidad para una mejor comprensión del tema ayudándoles; "a entender cuando leen, analizan y resuelven ejercicios reduciendo las complicaciones (dificultades para el estudiante) en detalles, que tal vez, a simple vista no vemos, además en cosas de geometría, con los videos les entendemos mejor" (Estudiante 81,90,69), en cambio el 58,8\% expresan que desconocen la utilidad de los programas en esta asignatura porque "no se ha mostrado ninguno, y por último, dichos programas son aburridos"(Estudiante 43), como se muestra en el cuadro No. 2: 
Cuadro No. 2: Los programas ayudan a entender más los temas recibidos

\begin{tabular}{|l|l|l|l|l|}
\hline \multicolumn{2}{|c|}{} & \multicolumn{2}{|c|}{$\begin{array}{c}\text { Consideras que los programas te ayudan a } \\
\text { entender más los temas recibidos en clases }\end{array}$} & Total \\
\hline \multirow{2}{*}{ Turno } & $\mathrm{Si}$ & $\mathrm{No}$ & $\mathrm{Si}$ \\
\cline { 2 - 5 } & Vespertino & 6 & 21 & 27 \\
\hline Total & Matutino & 34 & 36 & 70 \\
\hline
\end{tabular}

Los docentes exponen que "La forma en que se aborda el contenido en los textos escolares es desde el punto de vista de las aplicaciones, cuando deberían ser más dinámicos, más gráficos, orientando actividades claras sobre cómo enseñar o aprender las representaciones vectoriales, mediantes estrategias que se adecuen al medio donde interactúa el estudiante y donde se desarrollan de forma continua múltiples fenómenos los cuales se pueden representar no solo de forma Física si no también Matemática". Por lo que la utilidad de la biblioteca es esencial para los estudiantes de escasos recursos y para docentes al proporcionar los documentos necesarios para un estudio profundo de los contenidos abordados, permitiendo al estudiante acercarse al aprendizaje próximo, durante nuestra investigación y según los datos obtenidos, nos percatamos que el $92 \%$ de los estudiantes de décimo grado hacen uso de la biblioteca, de los cuales 27 estudiantes corresponden al turno vespertino y 65 al turno matutino; se agrega que los textos más utilizados son: "Física básica, Física general, El mentor de Matemática, Matemática de 4to año, Ciencias Físicas decimo y Enciclopedia general básica", a diferencia con el docente que utiliza por lo general para impartir la clase; "Física Tippens (general), Física de Héctor Pérez Montiel; Física, para ciencias e ingeniería Serway-Jewett 7 ma Ed. Vol. 1, y documentos de internet".

\section{Las dificultades en la enseñanza del Algebra de Vectores en Física de décimo grado}

Según el estudio realizado mediante las pruebas de Chi-cuadrado, cuadro No. 3, la percepción del estudiante en cuanto al grado de dificultad presentado por el contenido y la consideración de pertenencia (asignatura según el contenido) por el mismo, son altamente dependientes y necesarias para una mejor asimilación y desarrollo de los conocimientos, en los que los docentes afirman que "es necesario que el estudiante previo a la unidad debe saber algunos elementos de geometría; ángulos medidas, rotaciones, unidades para medir ángulos y el uso adecuado del estuche geométrico"; Empero sobre todo, agregan los docentes de Matemática: "el elemento más indispensable para estos estudiantes es saber graficar en el plano cartesiano y el dominio de reglas milimetradas y sus multiplillos a escala para poder interpretar las representaciones geométricas y algebraicas de diferentes situaciones (no sólo Física)". 
Cuadro No. 3: La percepción del estudiante en cuanto al grado de dificultad

\begin{tabular}{|l|r|r|r|}
\hline & \multicolumn{1}{|c|}{ Valor } & \multicolumn{1}{c|}{ gl } & Sig. asintótica (bilateral) \\
\hline Chi-cuadrado de Pearson & $22,844(\mathrm{a})$ & 6 &, 001 \\
\hline Razón de verosimilitudes & 17,706 & 6 &, 007 \\
\hline Asociación lineal por lineal & 8,963 & 1 & \\
\hline N de casos válidos & 97 & & \\
\hline $\begin{array}{l}\text { A } 7 \text { casillas (58,3\%) tienen una frecuencia esperada inferior a 5. La frecuencia mínima } \\
\text { esperada es ,31. }\end{array}$
\end{tabular}

Según los docentes de Matemática las actividades que se realizan durante el desarrollo del contenido "deben estar centradas en la ejemplificación de ejercicios concretos, explicando: composición de fuerzas, asociación de fuerzas, fuerzas equivalentes como si fuese estática de sólidos", de tal forma que las actividades propicien el desarrollo óptimo e integral de los contenidos, con respeto a las actividades que el docente realiza durante el período de clases, en la asignatura de Física y la información proporcionada por los estudiantes, se puede caracterizar que durante estas actividades desarrolladas por el docente es común $(6,2 \%)$ está centrada a la explicación de ejemplos Matemáticos, el 6,2\% se centra en la comparaciones del contenido con fenómenos Físicos, el 14\% para proporcionar ejemplos claros del tema y de interés para ellos, el 9\% de las actividades se desarrollan por medio de la utilización de textos de Física por el docente, el $2 \%$ el docente ha realizado otras actividades con los estudiantes como:

Cuadro No. 4: Actividades por el docente durante el desarrollo de la clase según los estudiantes

\begin{tabular}{|c|c|c|c|c|c|c|}
\hline \multirow{9}{*}{$\begin{array}{l}\text { Actividades } \\
\text { comunes por } \\
\text { el docente } \\
\text { durante el } \\
\text { desarrollo de } \\
\text { la clase }\end{array}$} & & \multicolumn{4}{|c|}{ Turno } & \multirow{3}{*}{ Total } \\
\hline & & \multicolumn{2}{|c|}{ Vespertino } & \multicolumn{2}{|c|}{ Matutino } & \\
\hline & & $\mathrm{Si}$ & No & $\mathrm{Si}$ & No & \\
\hline & Ejemplos de matemática & 9 & 18 & 21 & 49 & 97 \\
\hline & Comparaciones con fenómenos físicos & 10 & 17 & 20 & 50 & 97 \\
\hline & Ejemplos claros y de interés & 22 & 5 & 46 & 24 & 97 \\
\hline & Textos de física & 11 & 16 & 33 & 37 & 97 \\
\hline & Otros & 4 & 23 & 2 & 68 & 97 \\
\hline & Total & 56 & 79 & 122 & 228 & 485 \\
\hline
\end{tabular}

De el cuadro No. 4, también se deduce que sólo el 37,4\% de los estudiantes están pendientes de las actividades desarrolladas por el docente en pro del conocimiento del estudiante, por lo contrario el 79\% de los estudiantes no presta atención a las actividades que se desarrollan dentro del aula de clases, esto según los docentes se debe a "las dificultades más sentidas por los estudiantes que son: la falta de voluntad del estudiante durante las clases, el uso inadecuado del estuche geométrico, conceptos básicos y la falta de conocimientos de geometría en el estudiante". En cuanto a los 
contenidos desarrollados por el docente, explican que la dificultad más sentida no sólo para ellos, sino también repercute en el estudiante, radica en que los contenidos del programa deben reordenarse, de tal forma que se cumpla con la sistematicidad, pertenencia y profundidad de los temas y su relación al medio y necesidades futuras del estudiante.

Al solicitarles a los estudiantes que identificaran magnitudes vectoriales entre conceptos Físicos como: fuerza, distancia, tiempo, se comprobó que los estudiantes tienden a confundir los conceptos y características de los Vectores con los escalares, (gráfica No. 2) el 73\% de los estudiantes confundieron magnitudes escalares con magnitudes vectoriales. De los estudiantes que presentaron dificultades en la identificación de conceptos Físicos en magnitudes vectoriales y la comparación de los resultados obtenidos para identificar los elementos básicos se comprueba que el $83,3 \%$ de los estudiantes tienen dificultades al asociar los elementos básicos con los conceptos de fenómenos Físicos según sus características, en cambio, sólo el 16.6\% de los estudiantes demostró ser competente al asociar los elementos básicos de un Vector con los conceptos de fenómenos Físicos como magnitudes vectoriales.

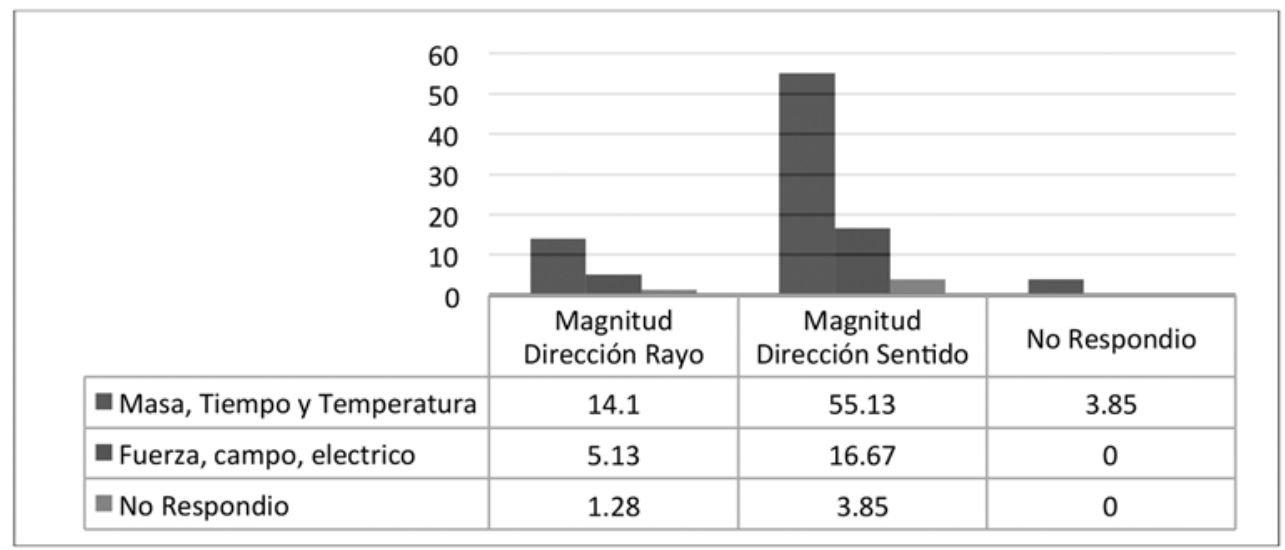

Figura No. 2: Identificación de los fenómenos que caracterizan de un Vector.

Una de las competencia de la unidad, en el programa de estudio de Educación Secundaria, es que el estudiante "representa gráficamente y a escala, situaciones de la vida real en donde se encuentra involucrada una magnitud vectorial y determina sus componentes" (Alvarado, 2011). Por lo que se le pidió a los estudiantes que identificaran la representación del Vector de un bote que navega 7 nudos al este y después 10 nudos al sur. Los resultados obtenidos demostraron que los estudiantes no utilizaron ni el método algebraico y mucho menos el método deductivo para la identificación adecuada de la representación de la situación expuesta, a pesar que se les brindó la gráfica de dicha situación, concluyendo que la mayoría de los estudiantes no son competentes para graficar situaciones Física y Vectores en el espacio, en cambio 15 de 78 estudiantes 
lograron ser competentes al identificar la representación de la situación, obviando el método algebraico y optaron por el método deductivo para darle solución al problema.

En cuanto al dominio de los conceptos de suma, la mayoría de los estudiantes desconocían el término de homogeneidad de la suma de magnitudes Físicas, demostrando en la mayoría de los casos no ser competentes para determinar que magnitudes se pueden sumar de forma vectorial, en la cual la minoría (10 estudiantes, entre ellos estudiante $5,18,34$ ) lograron identificar como homogeneidad al hecho de que una fuerza se puede sumar con otra, pero no con una velocidad. Pero en la operación de suma con componentes $\mathrm{i}, \mathrm{j}, \mathrm{k}$ expuesta en el ejercicio III. 1 de la prueba diagnóstica, solo tres de los estudiantes intentó solucionar la expresión que pedía sumar dos fuerza, la gravitatoria y una fuerza de impulso, desde sus componentes algebraicos, respetando el principio de homogeneidad, ambas situaciones son preocupantes debido a que se demuestra que aún cuando los docentes dedican gran parte del estudio a las operaciones algebraicas con Vectores, el estudiante no es capaz de realizar dichas operaciones con términos Físicos y mucho menos Matemáticos.

El propósito del problema "La velocidad de la corriente del río Plata es de $9 \mathrm{~km} / \mathrm{h}$, un campesino desea atravesar el río y nada perpendicular a la corriente a $5 \mathrm{~km} / \mathrm{h}$. ¿ $\mathrm{La}$ dirección que lleva el nadador es?" radicaba en el uso adecuado de las operaciones vectoriales, para la identificación de direcciones, como forma de introducción a los estudiantes a aplicaciones posteriores, este fue uno de los puntos de utilidad que expresaron la mayoría de los estudiantes, lo cual significaba que ejercía en el estudiante cierto grado de motivación, la forma de solución algebraica propuesta por los docentes es la deducción del módulo y posteriormente relacionarla con las funciones trigonométrica o aplicar la función tangente, en dicho ejercicio, solo 6 de los estudiantes establecieron el ángulo correcto a la situación expuesta, demostrando que la mayoría de los estudiantes no son capaces de deducir direcciones entre dos Vectores utilizando funciones trigonométricas y mucho menos algebraicas. Esta afirmación se comprueba al observar que los estudiantes no fueron competentes para proporcionar los datos solicitados en los últimos ejercicios de la prueba diagnóstica.

\section{Conclusiones}

- Se considera como una ventaja o valor agregado, que la disciplina está siendo desarrollada por docentes graduados en Ciencias de la Educación con mención en Matemática.

- Una de las dificultades o elementos que intervienen, es la visualización tradicional y abstracta del Álgebra de Vectores desde la perspectiva Matemática, sin profundizar en las aplicaciones Física, por parte del docente. 


\section{EDUCACIÓN}

- Los contenidos implícitos en la unidad de magnitudes escalares y vectoriales no se están desarrollando con la sistematicidad, pertenencia, profundidad acorde a las necesidades y saberes que pretende impulsar en los estudiantes el currículo de Educación Media y Básica en Nicaragua.

- Los estudiantes no son capaces de relacionar los conceptos aplicados a definiciones y fenómenos Físicos, haciendo uso inadecuado de propiedades del Álgebra vectorial durante los procedimientos aplicados, demostrando serias dificultades en cuanto a contenidos previos a la unidad.

- Las Estrategias Metodológicas utilizada por el docente no son las más adecuadas para la enseñanza de la unidad de Álgebra de Vectores implementada en Física, por ende en el desarrollo, los estudiantes demuestran falta de atención y voluntad para abordar de manera eficiente las asignaciones orientadas en período de clases.

- La motivación fomentada por los docentes, que busca mejorar la comprensión necesaria para hacer observaciones críticas y deducciones, no es significativa para el estudiante o carece de una explicación hacia donde se dirige dicha unidad.

- El uso excesivo del método deductivo por parte de los estudiantes repercute en la adquisición de un aprendizaje significativo del Álgebra de Vectores aplicada a Física.

- La insuficiencia y desactualización de los documentos proporcionados en la biblioteca a los estudiantes, con relación a los documentos utilizados por los docentes, necesarios para un estudio profundo de los contenidos abordados, no permiten al estudiante acercarse al aprendizaje próximo por medio del auto - estudio.

- Los docentes hacen uso de elementos TIC'S como medios visuales, obviando la verdadera importancia del aprendizaje por medio de la experimentación del ensayo-error, en el aula de clases.

\section{Lista de referencia}

Alvarado, P. O. (2011). Programa de estudios de educación secundaria, Física de 10mo y 11mo. Managua: MINED-Proyecto PASEN.

Etayo Miqueo, J. J., Garcí Heras, J. L., Ríos García, s., Ríos Insua, s., Santaló, L. A., \& Chica, Á. (1995). Enseñanza de las matemáticas en la educación secundaria (primera ed.). (V. G. Hoz, ed.) Álcalá, Madrid, España: Ediciones Rialp, S.A. 
Hernández, R., Fernández, C., \& Baptista, M. (2010). Metodología de la Investigación. México: McGraw-Hill.

Lupiáñez, J. L., \& Rico, 1. (2005). Análisis cognitivo en la formación inicial de profesores de matemáticas de secundaria. Oporto.

Lupiáñez, J. 1., \& Rico, L. (2006). Competencias y capacidades en el aprendizaje de los escolares. En J. L. Lupiáñez, \& L. Ric. Análisis didáctico y formación inicial (págs. 35-49). Bolea: M. J. González y M. Moreno.

MINED. (2009). Currículo Nacional Básico. Diseño curricular del subsistema de la educación básica y media nicaragüense. Pg. 62. Managua, Nicaragua: fondo nacionalproyecto pasen.

Moreno Bayardo, M. G. (200o). La enseñanza de la resolución de problemas matemáticos. El blanco y negro de algunas estrategias didácticas. Moreno B., M. (2000), la enseñanza de la resolución de problemas matemáticos, revistas de educación (Nueva Época. No. 159). 